\section{P443 ELIGIBILITY FOR AND USE OF HIV PRE-EXPOSURE PROPHYLAXIS AMONG AUSTRALIAN GAY AND BISEXUAL MEN OVER TIME}

${ }^{1}$ Garrett Prestage, ${ }^{1}$ Mohamed Hammoud, ${ }^{1}$ Benjamin Bavinton*, ${ }^{2}$ Adam Bourne, ${ }^{3}$ Martin Holt, ${ }^{4}$ Stefanie Vaccher, ${ }^{5}$ Louisa Degenhardt, ${ }^{1}$ Lisa Maher, ${ }^{1}$ Phillip Keen, ${ }^{1}$ Bridget Haire, ${ }^{6}$ Andrew Grulich, ${ }^{4}$ Fengyi Jin. ' UNSW Sydney, The Kirby Institute, Sydney, Australia; 'La Trobe University, Australian Research Centre in Sex Health and Society, Melbourne, Australia; ${ }^{3}$ UNSW Sydney, Centre for Social Research in Health, Sydney, Australia; ${ }^{4}$ The Kirby Institute, UNSW Sydney, Sydney, Australia; ${ }^{5}$ UNSW Sydney, National Drug and Alcohol Research Centre, Sydney, Australia; ${ }^{6}$ Kirby Institute, the University of New South Wales, Sydney, Australia

\subsection{6/sextrans-2019-sti.527}

Background Gay and bisexual men (GBM) increasingly use HIV Pre-exposure prophylaxis (PrEP) to prevent HIV infection. Eligibility for PrEP in Australia is based on behavioral criteria including methamphetamine use or condomless sex. It is unclear what proportions of GBM initiating PrEP meet these criteria over time.

Methods The Flux prospective cohort study enrolled Australian GBM between 2014 and 2018, following them every six months. We report PrEP use and behavioral eligibility for PrEP over time. Sexually transmissible infections data were not collected.

Results Among 1518 non HIV-positive men who were not using PrEP at baseline, mean age was 37.2 years (SD 13.13). Incident PrEP use increased from $2.8 \%$ at visit 2 to $11.9 \%$ at visit 6 (p-trend $<0.001$ ); eligibility for PrEP increased from $24.5 \%$ at baseline to $34.1 \%$ at visit 5 but fell to $20.4 \%$ at visit 6. Among all PrEP non-users, over one third were eligible for PrEP at some time during follow-up: $22.9 \%$ were eligible at visit 2; this proportion remained stable over subsequent visits $(21.5 \%$ at visit 5$)$ but fell to $9.6 \%$ at visit 6 . Less than $1 \%$ of PrEP users subsequently ceased use. Among continuing PrEP users, the proportion of non-eligible men remained steady at about $25 \%$ over time. Similar proportions (about 10\%) became eligible as ceased being eligible between visits.

Conclusion Although PrEP use or non-use was largely consistent with behavioral criteria for eligibility for its use, a substantial minority of GBM did not appear to use PrEP according to eligibility guidelines. About one-quarter of men who used PrEP were not eligible at the time while a similar proportion of PrEP non-users were eligible for its use, suggesting some underestimation of HIV risk. Greater efforts are needed to address these discrepancies between PrEP eligibility and its use, as engagement in risk behaviors changes over time.

Disclosure No significant relationships.

\section{P444 A CASE SERIES OF PRE-EXPOSURE PROPHYLAXIS FAILURES IN MEN USING EVENT-BASED-DOSING IN LONDON, UK}

${ }^{1}$ Naomi Fitzgerald*, ${ }^{2}$ Achyuta Nori. 'Guy's and St Thomas' NHS Trust, Burrell Street Sexual Health Clinic, London, UK; ${ }^{2}$ Guys' and St Thomas's NHS Foundation Trust, London, UK

\subsection{6/sextrans-2019-sti.528}

Background British guidelines recommend both event-based dosing (EBD) and daily dosing of combined tenofovir/emtricitabine (TDF/FTC) for HIV pre-exposure prophylaxis (PrEP) for men who have sex with men (MSM). Access to PrEP in the UK is either through a clinical study with limited capacity or by purchasing online. Dosing schedules are usually selfselected, often without access to professional advice or evidenced-based information. Non-daily dosing is popular as it is believed to be less toxic and more affordable than daily dosing.

Methods We describe three cases of HIV acquisition despite use of PrEP.

Results All were MSM and had a negative antigen/antibody HIV tests at 3 months of use. Case 1 switched to EBD after one month of daily PrEP. After several months he switched back to daily PrEP and had a positive HIV-1 antibody test six weeks later. Case 2 switched to EBD after 5 months of daily PrEP due to lower frequency of sex. He reported excellent adherence. He had a positive HIV-1 antibody test 4 months later. Case 3 was taking EBD PrEP for a total of 9 months. He had a positive HIV-1 antibody 5 months after his last negative HIV test. This was two months after his last episode of condomless sex. He reported occasional late dosing and some use of recreational drugs.

Conclusion It is likely that all three cases became infected while taking event-based PrEP. Two cases switched between daily and EBD due to lower self-perceived risk of HIV or frequency of sex. It is not clear if this may have affected PrEP efficacy. Evidence for effective EBD is strongly dependent on adherence and timing of doses. It is therefore vital that PrEP provision includes objective HIV risk assessment, adherence support and evidence-based dosing information to optimise efficacy.

Disclosure No significant relationships.

\section{P445 ANXIETY ABOUT HIV AND USE OF HIV PRE-EXPOSURE PROPHYLAXIS AMONG GAY AND BISEXUAL MEN}

${ }^{1}$ Garrett Prestage, ${ }^{1}$ Phillip Keen, ${ }^{1}$ Mohamed Hammoud, ${ }^{2}$ Adam Bourne, ${ }^{1}$ Benjamin Bavinton, ${ }^{3}$ Martin Holt, ${ }^{4}$ Stefanie Vaccher, ${ }^{5}$ Peter Saxton, ${ }^{1}$ Lisa Maher ${ }^{1}$ Bridget Haire, ${ }^{4}$ Fengyi Jin. 'UNSW Sydney, The Kirby Institute, Sydney, Australia; ${ }^{2}$ La Trobe University, Australian Research Centre in Sex Health and Society, Melbourne, Australia; ${ }^{3}$ UNSW Sydney, Centre for Social Research In Health, Sydney, Australia; ${ }^{4}$ The Kirby Institute, UNSW Sydney, Sydney, Australia; ${ }^{5}$ University of Auckland, School of Population Health, Auckland, New Zealand

\subsection{6/sextrans-2019-sti.529}

Background Many gay and bisexual men (GBM) experience anxiety about HIV, particularly in relation to sex. Use of HIV pre-exposure prophylaxis (PrEP) as an HIV prevention strategy may affect levels of HIV-related anxiety among GBM.

Methods Flux is an Australian online prospective study of GBM enrolled between 2014 and 2018. We measured anxiety using the generalized anxiety disorder assessment (GAD7) scale and a newly developed HIV anxiety scale developed using Principal Components Analysis. We assessed behavioral eligibility for PrEP based on Australian PrEP clinical prescribing guidelines. We used multivariate logistic regression to assess associations between use of PrEP and both GAD7 and HIVspecific anxiety.

Results Among 1574 men who completed the HIV anxiety scale, mean age was 37.2 years (SD 13.13). Men aged 25 years or younger had higher HIV anxiety scores than their older counterparts $(\mathrm{p}<0.001)$. Men who reported condomless anal intercourse with casual partners (CLAIC) scored higher on HIV anxiety than men who reported no CLAIC $(p=0.033)$. Among 1168 men who were not eligible for PrEP 
according to Australian guidelines, neither GAD7 nor HIV anxiety was independently associated with PrEP use. Among 406 PrEP-eligible men (26.2\%), PrEP users scored lower on GAD7 than did non-users $(\mathrm{OR}=0.95$; 95\%CI: 0.92-0.99) and PrEP use was independently associated with lower HIV anxiety $(\mathrm{aOR}=0.91 ; 95 \% \mathrm{CI}: 0.85-0.97)$.

Conclusion Among men who were eligible for PrEP, its use was independently associated with lower levels of anxiety in general, and of HIV anxiety specifically. PrEP use may help reduce anxiety among men who are at risk of HIV and may therefore offer perceived benefits in addition to avoiding HIV infection. This perceived benefit may be an important consideration in recommendations for PrEP use.

Disclosure No significant relationships.

\section{P446 USE OF ANTIBIOTIC PROPHYLAXIS FOR SEXUALLY TRANSMITTED INFECTIONS AMONG GAY AND BISEXUAL MEN IN AUSTRALIA}

${ }^{1}$ Vincent Cornelisse, ${ }^{2}$ Denton Callander, ${ }^{3}$ Christopher Fairley, ${ }^{4}$ Darren Russell. ${ }^{1}$ Melbourne Sexual Health Centre, Carlton, Australia; ${ }^{2}$ New York University, School of Medicine, New York, USA; ${ }^{3}$ Melbourne Sexual Health Centre, Melbourne, Australia; ${ }^{4}$ Queensland Health, Cairns Sexual Health Service, Cairns, Australia

\subsection{6/sextrans-2019-sti.530}

Background Antibiotic prophylaxis can reduce the risk of sexually transmitted infections (STIs), but concerns remain about its safety and feasibility of its implementation. We conducted an online survey to quantify current use of and interest in antibiotic prophylaxis among Australian gay and bisexual men. Methods From June to December 2018 our survey was promoted through gay community organisations and Melbourne Sexual Health Centre. The survey asked about demographics, sexual history, drug use, use of HIV pre-exposure prophylaxis (PrEP), history of STIs, use of antibiotic prophylaxis and attitudes towards antibiotic prophylaxis. We used logistic regression analyses to compare responses from respondents who had used antibiotic prophylaxis, respondents who had not used but were interested, and respondents who were not interested. Results A total of 517 survey responses were complete: 68 respondents $(13 \%)$ had previously used antibiotics to prevent STIs and $323(63 \%)$ expressed interest in using antibiotic prophylaxis. In univariate analyses, compared to respondents with no previous use and no interest, users of antibiotic prophylaxis were older $(M d n=43$ years vs $M d n=34$ years, $\mathrm{p}=0.018)$, had higher numbers of sexual partners $(M=14$ vs $M=5$, $\mathrm{p}=0.002)$, were more likely to report recent group sex $(64.7 \%$ vs $42.1 \%, \mathrm{p}=0.003)$, to be using HIV PrEP $(66.2 \%$ vs $35.7 \%, \mathrm{p}<0.001)$, and report a recent bacterial STI diagnosis $(57.4 \%$ vs $31.0 \%, \mathrm{p}<0.001)$. However, in multivariate analysis, users of antibiotic prophylaxis were not more likely to report a recent bacterial STI diagnosis $(\mathrm{aOR}=1.65,95 \% \mathrm{CI}$ 0.8-3.5), after adjusting for age, sexual partner numbers, drug use, and PrEP use.

Conclusion A majority of respondents expressed interest in antibiotic prophylaxis and $13 \%$ had used antibiotic prophylaxis. Users of antibiotic prophylaxis reported more STI risk factors and had more bacterial STIs than non-users. However, the use of antibiotic prophylaxis was not independently associated with a higher risk of STI diagnosis.

Disclosure No significant relationships.

\section{P447 AWARENESS AND ACCEPTABILITY OF PRE-EXPOSURE PROPHYLAXIS AMONG MSM: RESULTS FROM SCOTLAND'S GAY BAR SURVEY}

${ }^{1}$ Jamie Frankis*, ${ }^{2}$ Lisa Mcdaid, ${ }^{3}$ Lesley Wallace, ${ }^{4}$ David Goldberg, ${ }^{2}$ Paul Flowers. ${ }^{1}$ Glasgow Caledonian University, Glasgow, UK; ${ }^{2}$ University of Glasgow, MRC/CSO Social and Public Health Sciences Unit, Glasgow, UK; ${ }^{3}$ Health Protection Scotland, QU, UK; ${ }^{4}$ Health Protection Scotland, QE, UK

\subsection{6/sextrans-2019-sti.531}

Background Scotland was the first country to introduce free criterion-based PrEP for all citizens. So far, uptake has been almost exclusively among MSM. Herein, we examine PrEP awareness and acceptability among MSM in Scotland from a national level behavioural surveillance project.

Methods Time and location sampling was used to survey $\mathrm{n}=972$ MSM across the commercial gay scenes of Scotland's two largest cities.

Results $5.4 \%$ of participants were HIV+. 94.6\% were HIV-/ untested, of whom, $4.5 \%$ were on PrEP. Most of the remaining men had heard of PrEP (81.7\%). Multivariate logistic regression suggested that gay men $(\mathrm{OR}=2.76)$, men aged 26$35(\mathrm{OR}=2.38)$ and $36-45(\mathrm{OR}=2.31)$, men who used the gay scene $\geq=$ twice a month $(\mathrm{OR}=2.19)$, reported an HIV test in the last year $(\mathrm{OR}=1.96)$ or an STI other than HIV diagnosed in the last year $(\mathrm{OR}=3.47)$ were significantly $(p<0.05)$ more likely to have heard of PrEP. Around one third $(31.1 \%)$ of HIV-/untested men said they were likely to use PrEP now it is available. Multivariate logistic regression suggested that gay men $(\mathrm{OR}=2.23)$, younger men $(18-25$, $\mathrm{OR}=2.73 ; 26-35, \mathrm{OR}=2.48 ; 36-45, \mathrm{OR}=2.00)$, single men $(\mathrm{OR}=2.08)$, men who use the gay scene $\geq=$ twice a month $(\mathrm{OR}=1.51)$, men who report high risk condomless anal intercourse $(\mathrm{OR}=1.61)$, an HIV test in the last year $(\mathrm{OR}=1.95)$ or an STI other than HIV diagnosed in the last year $(\mathrm{OR}=1.61)$ were significantly more likely to consider using PrEP in the future.

Conclusion PrEP awareness among MSM in Scotland is high, but disparities in awareness remain along traditional indicators of inequality. Findings suggest substantial interest in PrEP, particularly among those most likely to benefit although other issues may be more indicative of use than pure behavioural risk. As the biobehavioural HIV risk management strategies now available to MSM require high levels of health literacy, health promotion strategies must focus on enabling men to negotiate these complexities.

Disclosure No significant relationships. 